\title{
Is Multipoint Pacing Superior to Optimized Single-Point Pacing?
}

\author{
Rodolfo San Antonio ${ }^{1}$, Jose Maria Tolosana ${ }^{2}$, and Luis Mont Girbau ${ }^{3}$ \\ ${ }^{1}$ Teknon Medical Centre \\ ${ }^{2}$ Hospital Clinic, Universitat de Barcelona; \\ ${ }^{3}$ Hospital Clinic de Barcelona
}

September 29, 2021

\begin{abstract}
Is Multipoint Pacing Superior to Optimized Single-Point Pacing?
Rodolfo San Antonio ${ }^{1}$, MD; Jose María Tolosana², MD, PhD; Lluís Mont ${ }^{2}, \mathrm{MD}, \mathrm{PhD}$.

${ }^{1}$ Heart Institute, Teknon Medical Center, Barcelona, Spain; University of Barcelona, Spain

${ }^{2}$ Institut Clínic Cardio-Vascular, Hospital Clínic, University of Barcelona, Spain; Institut d'Investigacions Biomèdiques August Pi I Sunyer (IDIBAPS), Barcelona, Spain; Centro de Investigación Biomédica en Red Enfermedades Cardiovasculares (CIBERCV), Madrid, Spain.
\end{abstract}

Word count: 434

*Corresponding Author:

Dr. Lluís Mont

Institut Clínic Cardio-Vascular, Hospital Clinic, Villarroel 170, 08036 Barcelona, Catalonia, Spain. Tel: +34 932275551.

Email:lmont@clinic.cat

\section{Funding: None}

\section{Disclosures:}

LM declares consulting services, and advisory boards to St. Jude Medical (Now Abbott), Medtronic, Biotronik, Boston Scientific, Livanova and Johnson\&Johnson. JMT declares consulting services, and advisory boards to St. Jude Medical (Now Abbott), Medtronic, Biotronik and Boston Scientific. RS declares no conflicts of interest with the subject matter discussed in the manuscript.

\section{Is Multipoint Pacing Superior to Optimized Single-Point Pacing?}

In this article, Mehta et al. ${ }^{1}$ add interesting data about the role of multipoint pacing (MPP) in cardiac resynchronization therapy (CRT). Despite the promising initial results, the largest randomized studies have failed to show a clear benefit of MPP in CRT. Therefore, the authors conclude that it is difficult to justify the widespread use of MPP in CRT.

Indeed, most MPP studies have focused on correcting intra-left ventricular dyssynchrony, recruiting more ventricular muscle than single-point pacing (SPP). However, these studies have neglected the potential role of fusion-based optimization with intrinsic conduction. In a recent study, we assessed the potential role of MPP in CRT by comparing MPP with SPP optimized by the fusion-optimized intervals (FOI) method. ${ }^{2}$ 
This method consists of achieving fusion between the paced rhythm and intrinsic conduction by modifying the atrioventricular (AV) and interventricular delays. We compared a single-point left ventricular pacing strategy optimized by FOI (SPP-FOI) with MPP programmed with a fixed AV interval of $130 \mathrm{~ms}$. The SPPFOI strategy resulted in greater QRS shortening than the MPP strategy $(-56 \mathrm{~ms}$ vs. $-42 \mathrm{~ms} ; \mathrm{P}<0.001)$. On the other hand, when FOI was added to MPP (MPP-FOI), there was no further shortening. Similarly, O'Donnell et al. ${ }^{3}$ observed that SPP with automatic AV delay optimization using the SyncAV algorithm resulted in a significant shortening of the QRS duration compared to non-optimized modes (SPP and MPP). Furthermore, they did not find a significant difference in QRS shortening when comparing SPP and MPP.

These two recent studies, not included in the previous review by Mehta et al., further reinforce the concept that most patients achieve their narrowest QRS when the AV interval is optimized to obtain fusion with intrinsic conduction, regardless of the use of MPP.

In summary, multipoint pacing was not superior to single-point pacing optimized with SyncAV or manual FOI, and MPP also resulted in higher battery drainage than SPP. It is possible to test individually whether MPP may be useful in specific cases by measuring the QRS duration with different options.

1. Mehta VS, Elliott MK, Sidhu BS, et al. Multipoint pacing for cardiac resynchronisation therapy in patients with heart failure: A systematic review and meta-analysis. J Cardiovasc Electrophysiol. 2021 Aug 11. doi: 10.1111/jce.15199.

2. San Antonio R, Guasch E, González-Ascaso A, et al. Optimized single-point left ventricular pacing leads to improved resynchronization compared with multipoint pacing. Pacing Clin Electrophysiol. 2021 Mar;44(3):519-527. doi: 10.1111/pace.14185.

3. O'Donnell D, Wisnoskey B, Badie N, et al. Electrical synchronization achieved by multipoint pacing combined with dynamic atrioventricular delay. J Interv Card Electrophysiol. 2021 Sep;61(3):453-460. doi: $10.1007 / \mathrm{s} 10840-020-00842-7$. 